\title{
Research on WVD of PCM/FM demodulation method
}

\author{
Yuan Binwen ${ }^{1}$, Wang $\mathrm{Yu}^{1}$, Chen Jian ${ }^{1}$, Fu Gang ${ }^{1}$ \\ ${ }^{1}$ China Satellite Maritime Tracking and Control Department, Jiangsu, Jiangyin, 214431, China
}

Keywords: Signal model, simulation analysis, noise, signal demodulation.

\begin{abstract}
PCM/FM signal model, this paper presents for the PCM/FM demodulation analysis presented PCM/FM signal characteristics of a new method -WVD law, and PCM/FM signal to be analyzed under different noise simulation, the results show , PCM/FM is a very effective method WVD analysis.
\end{abstract}

\section{Introduction}

PCM/FM telemetry as a current standard system, at home and abroad have a wide range of applications. With the rapid development of modern digital signal processing method and DSP technology, to achieve the PCM/FM signal is demodulated by the software method is not only possible, but also a trend in the development of the digital signal. Currently, PCM/FM signal demodulation software commonly used method is mainly based on the discrete short-time Fourier transform, the signal time-frequency analysis, the use of the use of a period of a sine curve model, sliding fitting method implementation PCM/FM precise demodulated signal ${ }^{[1-3]}$. The theoretical basis of the method is to assume that the signal in the window width is stationary. If you take too long each segment, the stationary hypothesis is not easy to set up, and the time domain resolution deteriorates the contrary, if the width of the window is too short to take the frequency domain resolution variation, which is a contradiction. If you can find a connection time and frequency bilinear transformation, so that the one-dimensional function of time or frequency function is mapped to the time - a two-dimensional function of frequency, and can accurately reflect the distribution of the signal energy over time, the frequency of the problem easier to solve, WVD transform adopted as this issue is resolved has opened up a new way.

WVD is an effective tool for analyzing the varying signal for detection and analysis of nonstationary time-varying signal, it is capable of non-stationary signal having a single frequency for precise frequency measurement. In this paper, the theory WVD frequency measurement is based on the characteristics of PCM / FM signal, the introduction of software demodulation principle and demodulation process and its performance analysis and simulation.

\section{PCM / FM signal}

Non Return to Zero pulse code modulation / frequency modulation (NRZ PCM/FM) is a hybrid coding and modulation scheme, in telemetry applications sometimes also called PCM/FM signal is continuous phase frequency shift keying (CPFSK). This mode is moved by the carrier wave representing a high frequency binary symbols "1" state, the carrier frequency represents a lower moved binary symbol "0" state. The mathematical expression is:

$$
S_{F M}(t)=A \cos \left[2 \pi f_{c} t+f_{d}+K_{F M} \int f(t) d t\right]
$$

Where in: A is the amplitude of the carrier; the carrier frequency; Providing Doppler frequency; as the modulation signal, the code rate is Rb ,; frequency offset constant. Symbol "1" and "0" corresponds to the carrier frequency are as follows:

$$
\begin{aligned}
& f_{1}=f_{c}+f_{d}+K_{F M} \times R_{b} \\
& f_{0}=f_{c}+f_{d}-K_{F M} \times R_{b}
\end{aligned}
$$


Therefore, within one symbol width, PCM / FM signal can be viewed as a single carrier modulated signal frequency. This can be applied to calculate the instantaneous frequency measurement method instantaneous frequency signal sampling points for the realization of the demodulated signal provided preconditions.

\section{WVD frequency measurement performance analysis}

By analyzing the first section, we can draw the following conclusions, demodulated PCM / FM signal is a carrier frequency of a frequency measurement; PCM / FM signal can be decomposed into two temporally single frequency carrier signal can be distinguished adding synthetic. Simulation analysis in PCM/FM signal frequency measurement of some of the features for the WVD algorithm. 3.1 WVD relatively simple frequency analysis signals and real signals

When the signal is a single frequency analytic signal $f(t)=A \exp \left(j \omega_{0} t\right) \quad-\infty<t<\infty$, according to the definition WVD can be drawn:

$$
W V D_{f}(t, \omega)=|A|^{2} 2 \pi \delta\left(\omega-\omega_{0}\right)
$$

I.e. WVD transform analytic signal of a single frequency for a single spectral line frequency axis. When the measured signal is a real signal of a single frequency, according to the definition can be drawn WVD:

$$
\left.\operatorname{WVD}_{f}(t, \omega)=|A|^{2} \frac{\pi}{2}\left\{\left[\delta\left(\omega-\omega_{0}\right)+\delta\left(\omega+\omega_{0}\right)\right]\right\}+2 \delta(\omega) \cos \left[2\left(\omega_{0} t+\varphi\right)\right]\right\}
$$

Visible, in addition to the normal frequency multiplier has a value of 2 as well as fluctuations in the external zero frequency, which is the interference cross terms.

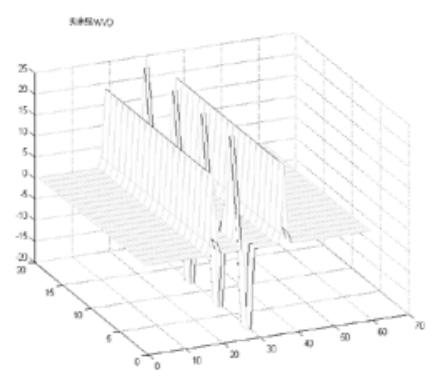

(a) WVD distribution analytic signal

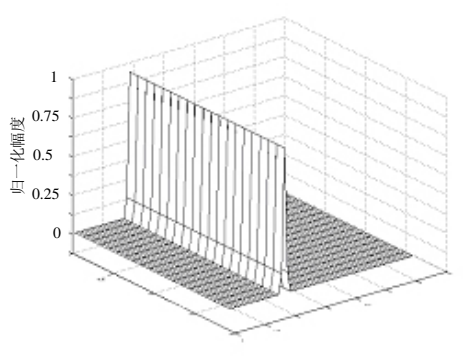

(b) WVD distribution of real signals

Single frequency analytic signal WVD conclusion, a single frequency without cross-term, there is a real signal WVD cross-term after a single frequency, both of which can be measured signal, the power measured after the analytical signal frequency WVD is a real signal of 4 times. WVD used to measure the frequency of PCM / FM signal, has affected the verdict if the cross-term, can be carried out WVD transform signal analysis.

\subsection{WVD distinguish real time two-frequency cosine signal and parsing WVD}

WVD real signals on a single frequency, including three, if it contains two frequencies, the situation will become more complicated, if the signal is not the same two frequencies can occur in the use of single-frequency signal analytical characteristics of WVD no cross-terms to improve WVD transform time-frequency result, more conducive to our judgment? after comparing the results shown in Figure 2 and parsing WVD WVD's real. 

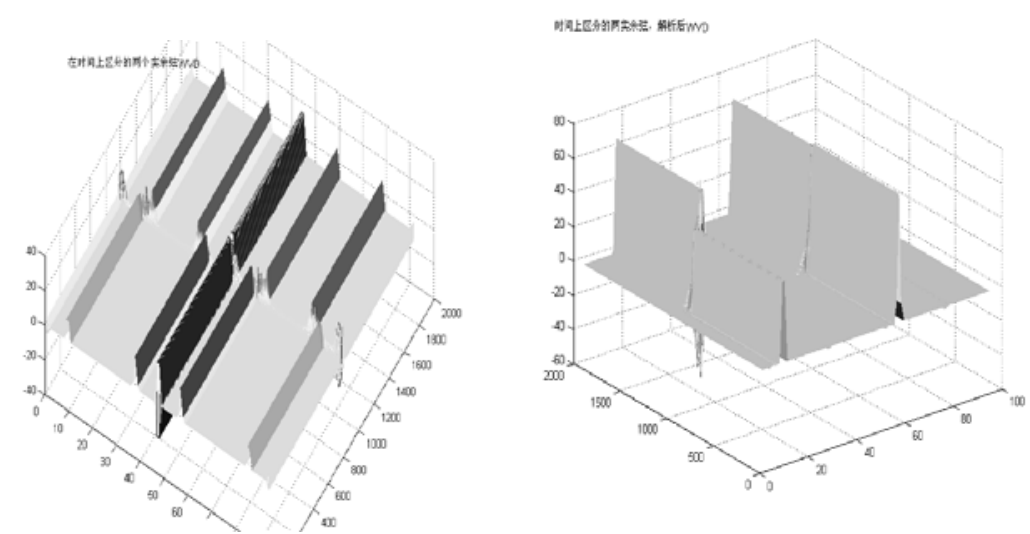

(a) WVD distribution of real signal of (b)WVD distribution analytic signal

Figure 2 After comparing real and parse the WVD

Conclusion by first parsing then WVD transform can effectively improve the coexistence of two frequencies simultaneously without signal frequency characteristics, energy is more concentrated, the judgment in favour of it at the expense of processing time for the price.

Comprehensive analysis of the above two points, WVD can be used to demodulate PCM/FM signal, due to the frequency concentration WVD well, so make sure to align the test WVD treatment frequency.

\section{Demodulation algorithm}

Demodulating the entire data can be divided into the following processes:

\subsection{Preliminary estimates signal parameters}

Because there is a deviation parameters with the theoretical value of the actual signal, in order to improve the accuracy of the demodulated signal is required before the data demodulation some preliminary estimates of the parameters. Parameter estimation are: sampling frequency fs, the sampling points within a single symbol Nc, 0 yards and one yard corresponding frequency $\mathrm{k} 0$ and k1.The first step: Use-by-point STFT (or instantaneous frequency measurement) method to get the signal f0 and $\mathrm{f} 1$ energy is relatively stable period of the signal (for high bit rate signals, this data should be greater than the length of $n$ equal to the width of two symbols; for low bit rate signals, one symbol width greater than or equal to). Instantaneous frequency measurement method at low signal to noise ratio is not applicable, it is recommended to use STFT. Step two: these n data as STFT, through certain steps (such as $5 \mathrm{KHz}$ ) k values of maximum energy at the point of the search, that is to get the true value of $\mathrm{k} 0$ and $\mathrm{k} 1$ are.

Third step: With the above parameters, and then demodulation processing the $\mathrm{N}$ data, $\mathrm{M}$ demodulated symbols. Then the average number of sampling points within each symbol, the same also amended the fs (under normal circumstances, the code rate is stable). In the demodulation process, due to the Doppler frequency, requires dynamic correction fs, especially at higher symbol rate case. In reality, fs is a fixed value, the reason for the existence of drift, due to changes in the Doppler frequency, performance in a changing number of sampling points. Thus, where fs is actually estimated in the estimated change of fd. Since fd is a slowly varying signal, has passed under the premise corrected if fs, the impact of the 64 yards Element fd will not bring the number of decoding errors, unless the rate of change of fd is very large, which is in practice does not exist. For example: the case where a code rate of $2 \mathrm{M}$, 64 consecutive cases even 1 , after the Doppler frequency has been corrected, the number of demodulation error still occurs, the average rate of change of fd is about $700 \mathrm{MHz} / \mathrm{s}$; $42 \mathrm{~K}$ code rate case, even 64 consecutive "1", the average change in the error rate fd $2.59 \mathrm{MHz} / \mathrm{s}$. But the scope of the contract changes to improve the $70 \mathrm{KHz}$, so, after the correction of the fs does not appear to change the number of demodulated symbols in the case of successive symbols. (I wonder if this analysis is reasonable) Some explanations:(1) there is no sampling methods for estimating the instantaneous frequency measurement frequency f0 or f1 in 
the current algorithm, mainly because of the circumstances under $10 \mathrm{~dB}$, frequency measurement accuracy is not guaranteed to achieve the correction of the results, which may also lead to greater deviation.(2) In the case of low bit rate, k value will affect the accuracy of the demodulation result, and not the size of fs for a single symbol sampling points of impact. Therefore, a smaller stepper unit, the value of $\mathrm{k}$ obtained high precision, then correction fs, the demodulated signal can be achieved.(3) In the case of high bit rate, the number of samples within a single symbol will directly lead to changes in the number of demodulated symbols. Therefore, we must first estimate fs. K values obtained by the method utilizing STFT not cause demodulation errors due to the frequency offset.

\subsection{Demodulated data}

The first step after the parameters to be estimated can begin demodulated.

Modulation frequency deviation determined in accordance with the known length of the sliding window, with STFT calculate signal k0 and k1 corresponding frequency spectrum, get the energy curve of the signal.

Compare the size of the two energy curve, the decision to generate double-edge signal, calculating the number of symbols of the two adjacent double edge between signals by the previous estimate of a single symbol of sampling points, solve codeword.

\subsection{Correction signal parameters}

Demodulation process correction is an adaptive process. After the completion of the original Amendment, in the demodulation process to dynamically correct the value of fs. The method is: after the first demodulation completion to give the average number of points within the symbol is $\mathrm{N} 1$, then when the second conducting demodulation, N1 used as the standard sample points, after the demodulation is completed, then corrected N1, N2 obtained. Correction algorithm is: Suppose the second demodulated symbols $m$ intervals $M$ samples of data, then the $N 2=(N 1+M) /(1+m)$; then, in turn amended N3, N4 ......, until end demodulation. The advantage is that before each demodulation, $\mathrm{N}$ use are the latest revised data, relatively close to the true value. Especially in the case for high speed continuous code symbol, can effectively suppress interference. If the decoding error Residue than 0.3 , it shows the parameters $\mathrm{k} 0$ and $\mathrm{k} 1$ at this time needs to be corrected. Correction method is to smooth the signal energy in the energy section of the search point corresponding to the maximum value of $\mathrm{k}$.

In order to analyze and resolve performance real WD, and the code rate is equal 2MBPS, sampling rate 20MSPS, peak frequency deviation is 0.35 times the symbol rate PCM/FM modulation signal "1" code power spectrum analysis simulation results, see Figure 4. The figure, the horizontal axis represents the sampling points, the longitudinal axis "a" code power spectrum estimation value.



Figure 4 real WD and parse for PCM/FM frequency measurement

In real WD, "1" code and the estimated value of the maximum noise power spectrum with the minimum gap between the 19, where the gap between 01 and 14 alternating. WD parsing, the "1" code and the estimated value of the maximum noise power spectrum with the minimum gap between the 55, where the gap between 01 and 40 alternating. According to the simulation result, 
when the high-WD method can be used, taking into account the processing speed problem, try to use the real-WD, WD in parsing the interference cross term severe use.

\subsection{Use WD (real signal) algorithm demodulation process}

WD algorithm is based on the instantaneous autocorrelation function before converting to data within the data window seek instantaneous autocorrelation.

\subsection{Use WD (analytical signal) algorithm demodulation process}

WD parsing algorithm for demodulation, on the basis of real WD algorithm, before conversion required data within the data window to obtain the corresponding analytical signal (by Hilbert transform completed).
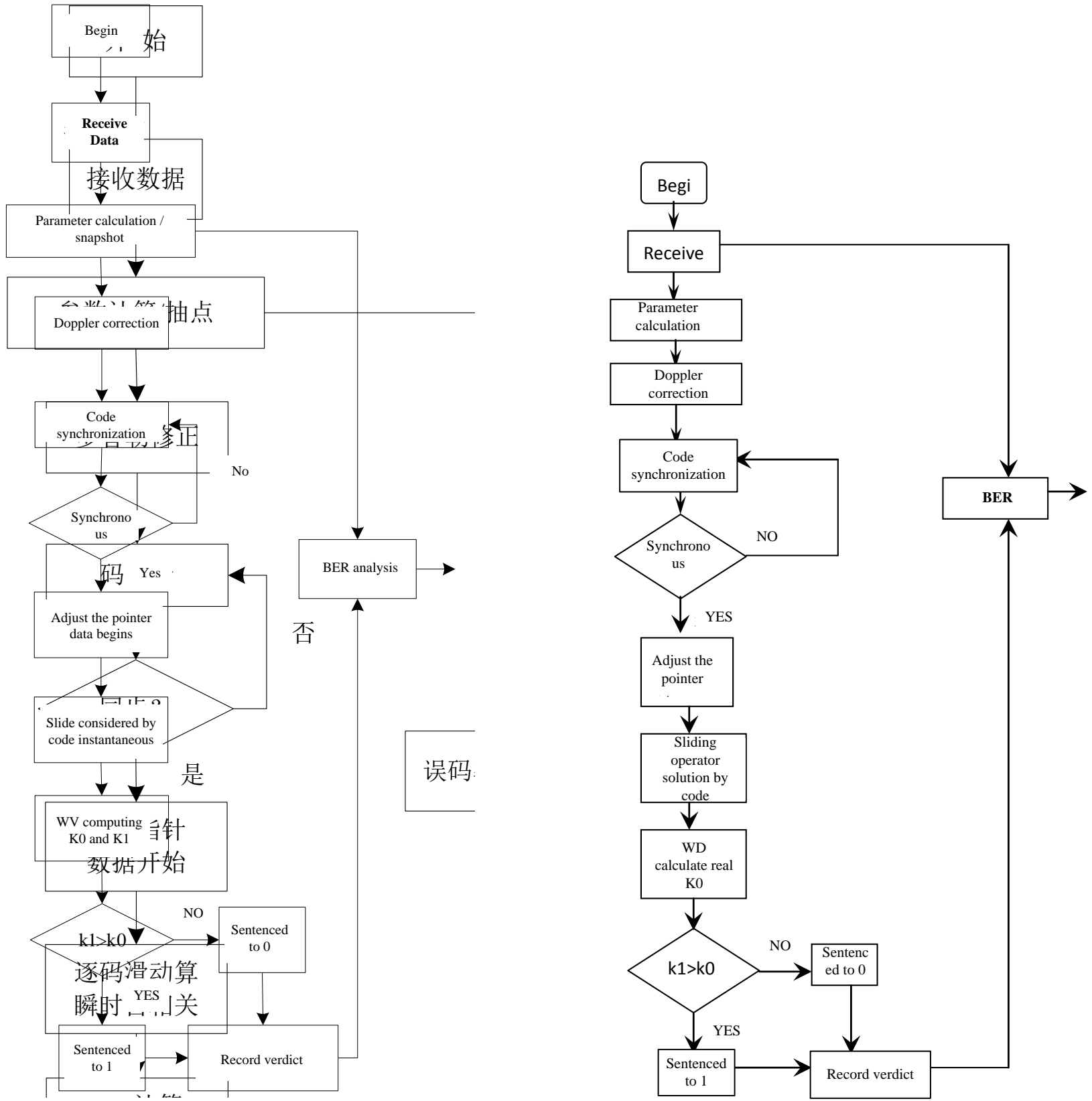

Figure 5 WD real demodulation algorithm simulation flow chart algorithm simulation flow chart parsing

\section{References}

[1] Stackelberg H. The Theory of the Market Economy. Oxford: Oxford University Press, 2012.

[2] Miller,T., Friesz,T. \& Robin,R., Heuristic algorithms for delivered pric spatially competitive network facility location problem. Ann. Oper. Res., 34,pp.177-202,2012. 
[3] Pieume, C.O, Fotso L.P. \& P. Siarry. A method for solving bilevel linear progremming problem. Journal of Information and Optimization Science, 29(2), pp. 335-358,2008.

[4] Aryanezhad,M.B. \& Roghanian, E.A., Bilevel linear multi-objective decision making model with interval coefficents for suppy chain coordination.Iust Internation Journal of Engineering Science, 19(1-2), pp.67-74,2008,.

[5] Cheng-Min Feng \& Chieh-chao Wen, A fuzzy bi-level and multi-objective model to control traffic flow into the disaster area post earthquake. Journal of the Eastern Asia Society for Transportation Studies,6,pp.4253-4268,2005.

[6] Li He-Cheng, Wang Yu-Ping, An interpolation based genetic algorithm for sloving nonlinear bilevel programming problems.Chinese Journal of Computers, 31(6), pp.910-918, June 2008.

[7] Kalyanmoy Deb, Amrit Pratap, Sameer Agarwal \& Meyarivan, T.A., Fast and elitist multiobjective genetic algorithm: NSGA-II, IEEE Trans. Evol. Comp., 6(2), pp.182 - 197,2012.

[8] Wang Yu-Ping, The Theory and Method of Evolutionary Computation, Beijing: Science Press,2011. 